\title{
BIOFILMS
}

\section{Biofilms take shape}

A recent paper in Proceedings of the National Academy of Sciences USA confirms the importance of bacterial quorum sensing-controlled surfactants in biofilm structuring and detachment.

The 'lifecycle' of a biofilm involves several distinct stages, from attachment, to maturation, to detachment and dispersal. Michael Otto and colleagues were interested in the factors

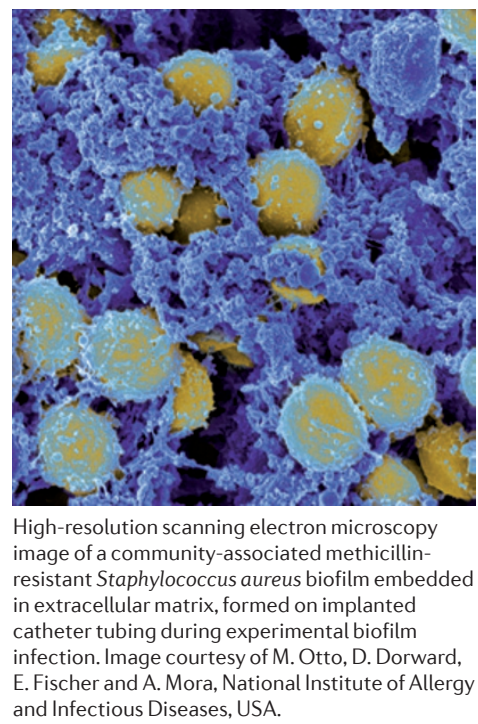

involved in the development of the structure of a mature Staphylococcus aureus biofilm and its eventual detachment. Although the specific factors involved in biofilm detachment in S. aureus are unknown, the overall process is known to be regulated by the Agr quorum sensing system. Moreover, recent work in the related organism Staphylococcus epidermidis revealed a role for a specific class of phenol-soluble modulins (PSMs) - small $\alpha$-helical, amphipathic peptides with surfactant qualities - in biofilm detachment.

S. aureus encodes a total of nine PSMs. The authors created a series of isogenic psm mutants and then, using confocal laser scanning microscopy, undertook a comprehensive analysis of the structure of the biofilms formed by the psm mutants and an agr mutant in both static and dynamic (flow cell) conditions. The results showed that in both static and dynamic biofilms the agr and psm mutants elaborated smoother biofilms with poorer channel formation and greater thickness and volume than the biofilms formed by wildtype $S$. aureus, indicating a role for
Agr and PSMs in biofilm structuring and detachment. Analysis of the patterns of agr and psm expression within dynamic biofilms showed that expression varied both with time and with position in the biofilm, and suggested that Agr controls PSM production. Finally, in a mouse catheterassociated infection model, a triple psm deletion strain showed reduced dissemination to the lymph nodes, skin and the tissues surrounding the catheter.

This work confirms that PSMs have a key role in structuring and detachment of S. aureus biofilms. Given that similar quorum sensingcontrolled surfactants are encoded by many other biofilm-forming bacterial species, the authors speculate that this represents a widespread mechanism of controlling biofilm structure and detachment.

Sheilagh Molloy

ORIGINAL RESEARCH PAPER Periasamy, S. et al. How Staphylococcus aureus biofilms develop their characteristic structure. Proc. Natl Acad. Sci. USA 109, 1281-1286 (2012)

FURTHER READING McDougald, D. et al. Should we stay or should we go: mechanisms and ecological consequences for biofilm dispersal. Nature Rev. Microbiol. 10, 39-50 (2012) 